\title{
Antioxidant activity of fungi isolated from soil of different areas of Punjab,India
}

\author{
Priyanka Chandra and Daljit Singh Arora* \\ Microbial Technology Laboratory, Department of Microbiology, Guru Nanak Dev University, Amritsar-143005, INDIA \\ *Corresponding author. E-mail: daljit_02@yahoo.co.in
}

\begin{abstract}
The study was carried out to investigate the antioxidant activity of fungi isolated from soil of different areas of Punjab, India and compare their efficiency with a known antioxidant, ascorbic acid. The antioxidant potential of fungal extracts was quantified by DPPH and reducing power assay. Total phenolic contents were estimated using Folins-Ciocalteau (FC) reagent. Out of 113 fungal isolates selected, 51 were having antioxidant potential and these were further assayed quantitatively. All of these showed good activity against DPPH radical while 32 of these isolates demonstrated reducing potential also. In addition, some were equally good as ascorbic acid. The present study demonstrated potential of soil fungi to have antioxidant activity similar to plants and mushrooms. High phenolic content of fungi further highlight their significance as new sources of natural antioxidants. These fungi may provide easier set up for production and purification of natural antioxidants as compared to higher plants.
\end{abstract}

Keywords: Antioxidant activity, Dot-blot DPPH staining, DPPH assay, Reducing power, Soil fungi

\section{INTRODUCTION}

In the past few years, there has been growing interest in the reactive oxygen species (ROS) due to their involvement in several pathological situations (Song and Yen, 2002). Reactive oxygen species (ROS) include, superoxide anion $\left(\mathrm{O}_{2}^{-}\right)$and alkoxyl (RO) radical, nitric oxide (NO), hydrogen peroxide $\left(\mathrm{H}_{2} \mathrm{O}_{2}\right)$, peroxyl radical (ROO) and hypochloride ( $\mathrm{HOCl})$. Superoxide anion radical $\left(\mathrm{O}_{2}^{-}\right)$and hydrogen peroxide $\left(\mathrm{H}_{2} \mathrm{O}_{2}\right)$ can interact in the presence of certain transition metal ions to yield a highly reactive oxidizing species, the hydroxyl radical $\left(\mathrm{OH}^{\cdot}\right)$ (Valentao et al., 2002).

The oxidation induced by ROS may result in cell membrane disintegration, membrane protein damage and DNA mutations which play an important role in aging and can further initiate or propagate the development of many diseases, such as arteriosclerosis, cancer, diabetes mellitus, liver injury, inflammation, skin damages, coronary heart diseases and arthritis (Moon et al.,2006, Tiwari and Tripathi, 2007 ). Although, the body possesses such defense mechanisms, as enzymes and antioxidant nutrients that arrest damaging properties of ROS (Virtanen et al., 2007) however, their prolonged exposure may lead to irreversible oxidative damage (Tiwari and Tripathi, 2007). Therefore, antioxidants with free radical scavenging activities may have great relevance in the preservation and therapeutics of diseases involving oxidants or free radicals (Zhao et al., 2006). The antioxidants serve as a defensive factor against free radicals in the body. Enzymes such as superoxide dismutase, catalase and glutathione peroxidase are the main enzymes that oppose oxidation. If the free radical production becomes more than the capacity of enzymatic system to cope up with, then the second line of defense (vitamins) may come to rescue. Vitamin $\mathrm{A}$ and $\mathrm{C}$ quench free radicals by oxidizing and inactivating them. The polyphenolic compounds commonly found in plants, mushrooms, and fungi have been reported to have multiple biological effects such as anti-inflammatory, antiarteriosclerotic, antitumor, antimutagenic, anticarcinogenic, antibacterial and cardio protective actions including antioxidant activity (Miller et al., 1995). Various natural resources including plants, fungi and mushrooms have been known to produce many biologically active substances including potent antioxidants. Such natural antioxidants have already been isolated from plants and mushrooms but production and downstream processing of actual bioactive phytochemicals and secondary metabolites is quite tougher as compared to microbes. Various microorganisms, such as bacteria, fungi, and actinomycetes are rich sources of bioactive molecules, which have been used for the treatment of various human infectious diseases. However, a vast biodiversity remains untapped that can be exploited for the benefit of humankind. A variety of fungal strains such as Antrodia camphorata (Song and Yen, 2002); Penicillium roquefortii, Aspergillus candidus, Mortierella, Emericella falconensis, Acremonium, Colletotrichum gloeosporioides (Rios et al., 2006), Mycelia sterilia (Moon et al., 2006), Chaetomium sp., Cladosporium sp., Torula sp., Phoma sp. (Huang et al., 2007) etc. have been reported to produce novel antioxidants. The present study 
was planned to investigate the antioxidant activity of some soil fungi isolated from various geographical areas of Punjab, India and compare them with ascorbic acid, a known antioxidant.

\section{MATERIALS AND METHODS}

Isolation and growth of fungi: Fungi were isolated from soils of different regions of Punjab. The fungal isolates so obtained were identified on the basis of morphology of the fungal culture, characteristics of the spores. The cultures were also preserved in $10 \%$ glycerol at $-70^{\circ} \mathrm{C}$. The isolated fungi were grown on YGA plates for 6-7 days from which two discs $(8 \mathrm{~mm})$ of fungal mycelia were used to inoculate $15 \mathrm{ml}$ of Czapek dox's broth in $50 \mathrm{ml}$ tubes and incubated in an incubator at $25^{\circ} \mathrm{C}$. After 10 days incubation, the culture broth of each fungus was filtered through Whatmann filter paper no.1 and the filtrate so obtained was used for different assay procedures.

\section{Rapid screening for antioxidant activity by dot-blot DPPH} staining: Initially, all the 113 fungi isolated from soil were screened for their antioxidant activity according to dot blot DPPH rapid staining method (Huang et al., 2006). Drops taken from culture broth were spotted onto a TLC plate (Silica gel $60 \mathrm{~F}_{254}$ ) with the help of micro capillary and allowed to dry for $3 \mathrm{~min}$. The staining of silica gel TLC plates was carried out by placing them upside down for $10 \mathrm{sec}$ in $0.4 \mathrm{mM}$ DPPH solution. The excess solution was removed with a blotting paper and the plates were air-dried. Stained silica layers revealed a purple background with white spots indicating radical scavenging capacity. The intensity of the white color depends upon the amount and nature of radical scavengers present in the sample. Ascorbic acid was taken as the standard and distilled water was taken as negative control.

\section{Quantitative assay for DPPH free radicals scavenging} activity:The scavenging activity for DPPH free radicals was measured according to Zhaoet al. (Zhaoetal., 2006). An aliquot of $1 \mathrm{ml}$ of $0.1 \mathrm{mM}$ DPPH solution in ethanol and $0.5 \mathrm{ml}$ of extract were mixed. The mixture was shaken vigorously and allowed to reach a steady state at room temperature for $30 \mathrm{~min}$. Decolourization of DPPH was determined by measuring the absorbance at $517 \mathrm{~nm}$, and the DPPH radical scavenging was calculated according to the following equation:

$\%$ scavenging rate $=(1-(\mathrm{A} 1-\mathrm{A} 2) / \mathrm{AO}) \times 100$,

where $\mathrm{AO}$ was the absorbance of the control and A1 was the absorbance of extract, A2 was the absorbance without DPPH. Ascorbic acid was taken as the standard. All the tests were performed in triplicate.

Determination of antioxidant activity by reducing power measurement: The reducing power of the extracts was determined according to Changet al. (Chang etal., 2002). An aliquot of $0.5 \mathrm{ml}$ extract was added to $0.1 \mathrm{ml}$ of $1 \%$ potassium ferricyanide. The mixture was incubated at 50 ${ }^{\circ} \mathrm{C}$ for $30 \mathrm{~min}$, during which period ferricyanide was reduced to ferrocyanide and supplemented with $0.1 \mathrm{ml}$ of $1 \%$ trichloroacetic acid and was mixed with $0.1 \% \mathrm{FeCl}_{3}$, and left for $20 \mathrm{~min}$. Absorbance was read at $700 \mathrm{~nm}$ to determine the amount of ferric ferrocyanide (Prussian blue) formed. Increased absorbance of the reaction mixture indicated increased reducing power of the sample. Ascorbic acid was taken as the standard.All the tests were performed in triplicate.

Determination of total polyphenolic content (TPC): The total polyphenolic contents were determined colorimetrically using the Folin-Ciocalteau (FC) method according to Singleton et al. (Singleton et al., 1999) with some modifications. Test sample $(0.5 \mathrm{ml})$ was mixed with $0.2 \mathrm{ml}$ of FC reagent and allowed to stand for $10 \mathrm{~min}$ to which $0.6 \mathrm{ml}$ of $20 \%$ sodium carbonate was added and mixed completely. The reaction mixture was incubated at $40^{\circ} \mathrm{C}$ for $30 \mathrm{~min}$. Absorbance of the reaction mixture was measured at $765 \mathrm{~nm}$. Gallic acid was taken as standard. All the tests were performed in triplicate.

Determination of Mycelial biomass: The dry weight of mycelia was measured after repeated washing of the mycelial pellets with distilled water and drying overnight at $70^{\circ} \mathrm{C}$ to a constant weight.

Statistical analysis: The results are expressed as mean \pm SD values. Pearson's correlation coefficient was also analysed. $p$ value less than 0.05 were considered statistically significant.

\section{RESULTS}

Fungal isolates: Majority of the fungal isolates (23) belonged to the genus Aspergillus while 17 isolates were identified as Penicillium. In addition, one genus was identified as Rhizopus while rest of the fungal isolates could not be identified.

Rapid screening of antioxidant activity by dot blot assay: Antioxidant capacity of fungal extracts was detected semi-quantitatively by a rapid DPPH staining method. This method is typically based on the inhibition of the accumulation of oxidized products, since the generation of free radicals is inhibited by the addition of antioxidants and scavenging of the free radicals shifts the end point. The appearance of a white color spot on a purple background has potential value for the indirect evaluation of antioxidant capability of the each fungus in the dot blots. Out of 113 fungal isolates, 51 showed white colored spot against purple background (Fig. 1). The intensity of white color was however variable for different organisms. These positive strains were selected for further studies and quantification.

Quantitative assay for DPPH free radicals scavenging activity: DPPH molecule that contains stable free radicals has been widely used to evaluate the radicals scavenging ability of antioxidants. The reduction ability of DPPH 


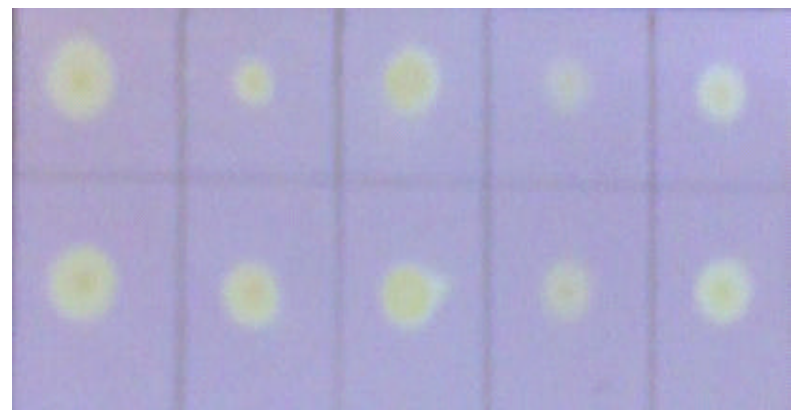

Fig. 1. Dot blot qualitative assay of some positive fungal extracts for antioxidant activity.

radical formation was determined by the decrease in its absorbance at $517 \mathrm{~nm}$ induced by antioxidants. All the positive fungal strains (51) used for quantitative studies showed good activity. The Aspergillus isolates PR 15, PR 38, PR 70 and Penicillium PR 46 showed the highest DPPH scavenging activity ranging from $80 \%-92 \%$. Ascorbic acid as standard showed $96.87 \%$ scavenging activity. Aspergillus PR 40, PR 57, and Rhizopus PR 61 gave relatively poor performance $(27 \%-39 \%)$. (Table. 1). The control broth without fungal extract showed no activity.

Determination of antioxidant activity by reducing power measurement: Reducing power measure the ability of a sample to act as an electron donor and therefore, reacts with free radicals converting them to more stable products and thereby terminate radical chain reaction. In order to examine the reducing power of fungal extracts, the reduction of $\mathrm{Fe}^{3+}$ to $\mathrm{Fe}^{2+}$ was observed. Of the 51 positive strains selected as above, 32 showed reducing power to variable extent and some of the isolates viz Aspergillus PR 64, PR 78,PR 66 and Penicillium PR 46 showed even higher reducing power than ascorbic acid (Table 1). The control i.e. broth without fungal extract showed no activity.

Total phenolic content (TPC) and biomass: The total phenolic content of fungal extracts was determined using FC reagent. The highest TPC was observed in Penicillium PR 46 which contained $20.59 \mathrm{mg} / \mathrm{ml}$ of filtrate and was followed by Aspergillus PR 15, PR 64, PR 66, PR 78, PR 113 varying from $11-19 \mathrm{mg} / \mathrm{ml}$ of filtrate, while Rhizopus PR 61, PR 16,PR 19 had the lowest amount of phenolic compounds with $1.47,1.19,1.02 \mathrm{mg} / \mathrm{ml}$ of filtrate, respectively. The media used for growth of fungi did not show any phenolic content. The fungal biomass obtained at the end of incubation period of 10 days for different fungi ranged from 93 to $223 \mathrm{mg}$ (Table 1).

\section{DISCUSSION}

Epidemiological studies show that human body is damaged by reactive oxygen and nitrogen species. Thus, it is considered important to increase the intake of antioxidants in the human diet. Some synthetic antioxidants may exhibit toxicity with carcinogenic potential, show lower efficiency than natural antioxidants, and require high manufacturing costs. Thus, there is a need to identify natural and possibly more economic and effective antioxidants (Mathew and Abraham, 2006; and Kumar and Chattopadhaya, 2007). Natural antioxidants are also in high demand for application as nutriceuticals as well as food additives because of consumer's preferences. (Zielinski and Kozlowska, 2000; and Lee and Yen, 2006). In the present study, 45 percent of soil fungal isolates possessed antioxidant activity and most of them belonged to genus Penicillium and Aspergillus. Qualitative test proved successful for bulk screening of samples though quantitative analysis revealed the differential ability of such fungi. On the basis of qualitative test all the short listed fungal strains were positive for DPPH free radical scavenging activity and the different organisms revealed variable antioxidant potential, which may be due to neutralization of free radical character of purple color DPPH either by transfer of electron or hydrogen atom to yellow colored diamagnetic molecule (Bounatirou et al.,2007). The reducing power evaluation of the test compounds is an important parameter related to assessing the antioxidant activity. The extracts act as reductones that inhibit lipid peroxidation by donating a hydrogen atom thereby terminating the free radical chain reaction. Out of 51 fungi positive for DPPH free radical scavenging activity, 32 were found to possess reducing potential, which may be due to the di-monohydroxy substitution in the aromatic ring, which possesses potent hydrogen donating ability (Chang et al., 2002).

Previous studies reveal phenolic compounds to be major antioxidants of medicinal plants, mushrooms, essential oils, spices, fruits and vegetables (Mokbel and Hashinaga, 2006). The interest in the phenolic compounds has increased tremendously due to their prominent free radical scavenging activity (Robbins.,2003), attributed to their redox properties, which allow them to act as reducing agents or hydrogen atom donor (Amarowicz et al.,2004).The importance of phenolic contents has been endorsed from the present observation of their high content in soil fungi. Aspergillus PR 78, PR64, PR66, and Penicillium PR46 possessed high phenolic content with good reducing power as well as DPPH scavenging activity. Some studies have proved that the phenolic substances such as flavonoids and phenolic acids are considerably more potent antioxidants than vitamin $\mathrm{C}$ and vitamin E. These compounds have also been found to exhibit many other health related properties because of their antioxidant activities (Aljadi and Kamaruddin, 2004).

The comprehensive evaluation of antioxidant activity of natural products using different tests has been shown to be important in assessing antioxidant activity of endogenous compounds. It is attractive for researchers 
Table 1. Antioxidant potential of soil fungi.

\begin{tabular}{|c|c|c|c|c|}
\hline Strains & DPPH assay $^{\text {a }}$ & Reducing power & $\begin{array}{l}\text { Total phenolic } \\
\text { content }^{b}\end{array}$ & Biomass $^{c}$ \\
\hline Aspergillus $\mathrm{PR} 2$ & $70.32 \pm 0.15$ & - & $2.5 \pm 0.001$ & $196 \pm 0.001$ \\
\hline Aspergillus PR 3 & $63.74 \pm 0.76$ & - & $4.14 \pm 0.030$ & $204 \pm 0.110$ \\
\hline Aspergillus PR 4 & $74.29 \pm 0.20$ & $0.673 \pm 00$ & $6.95 \pm 00$ & $177 \pm 0.030$ \\
\hline PR 5* & $67.22 \pm 0.54$ & $0.115 \pm .0005$ & $2.98 \pm 0.050$ & $98 \pm 0.0010$ \\
\hline Aspergillus $\mathrm{PR} 7$ & $69.15 \pm 0.18$ & $0.153 \pm 0.001$ & $3.76 \pm 0.02$ & $180 \pm 0.009$ \\
\hline PR $13^{*}$ & $70.27 \pm 0.27$ & - & $4.86 \pm 0.02$ & $193 \pm 0.006$ \\
\hline Aspergillus PR 15 & $85.69 \pm 0.23$ & $0.768 \pm 0.001$ & $15.46 \pm 0.002$ & $173 \pm 0.002$ \\
\hline PR 16* & $66.42 \pm 0.41$ & $0.202 \pm 0.001$ & $1.19 \pm 0.30$ & $93 \pm 0.004$ \\
\hline Penicillium PR 17 & $74.60 \pm 1.50$ & $0.5 \pm 0.005$ & $8.53 \pm 0.30$ & $201 \pm 0.007$ \\
\hline Aspergillus PR 18 & $78.10 \pm 0.36$ & $0.485 \pm 00$ & $4.98 \pm 0.030$ & $205 \pm 0.007$ \\
\hline Penicillium PR 19 & $45.26 \pm 0.26$ & - & $1.01 \pm 0.02$ & $102 \pm 0.001$ \\
\hline Aspergillus $\mathrm{PR} 20$ & $74.52 \pm 0.37$ & - & $4.19 \pm .040$ & $169 \pm 0.009$ \\
\hline Aspergillus PR 22 & $85.91 \pm 0.27$ & $0.136 \pm 0.001$ & $3.98 \pm 0.03$ & $150 \pm 0.009$ \\
\hline Aspergillus PR 23 & $80.09 \pm 0.10$ & - & $3.157 \pm 0.01$ & $202 \pm 0.006$ \\
\hline Aspergillus $\mathrm{PR} 26$ & $74.46 \pm 0.31$ & - & $2.77 \pm 0.030$ & $172 \pm 0.003$ \\
\hline Penicillium PR 28 & $75.01 \pm 0.45$ & $0.567 \pm 0.001$ & $7.1 \pm 0.10$ & $183 \pm 0.001$ \\
\hline Aspergillus PR 38 & $92.42 \pm 0.21$ & $0.530 \pm 0.001$ & $5.6 \pm 0.001$ & $163 \pm 0.006$ \\
\hline Penicillium PR 40 & $27.13 \pm 0.478$ & - & $9.68 \pm 0.08$ & $200 \pm 0.002$ \\
\hline Penicillium PR 42 & $67.44 \pm 0.82$ & $0.634 \pm 0.002$ & $9.69 \pm 0.98$ & $206 \pm 0.002$ \\
\hline Aspergillus $\mathrm{PR} 43$ & $70.18 \pm 0.10$ & - & $2.67 \pm 0.02$ & $208 \pm 0.030$ \\
\hline Aspergillus $\mathrm{PR} 45$ & $70.33 \pm 0.09$ & - & $4.62 \pm 0.001$ & $180 \pm 0.010$ \\
\hline Penicillium PR 46 & $81.65 \pm 0.054$ & $1.08 \pm 00$ & $20.59 \pm 0.1$ & $195 \pm 0.008$ \\
\hline Penicillium PR 49 & $70.53 \pm 0.30$ & $0.780 \pm 0.002$ & $9.52 \pm 0.21$ & $171 \pm 0.001$ \\
\hline Penicillium PR 53 & $65.22 \pm 0.09$ & $0.642 \pm 0.008$ & $6.38 \pm 0.24$ & $202 \pm 0.009$ \\
\hline Penicillium PR 57 & $39.02 \pm 0.51$ & - & $1.1813 \pm 0.08$ & $163 \pm 0.009$ \\
\hline Penicillium PR 60 & $58.51 \pm 1.02$ & $0.561 \pm 0.009$ & $6.68 \pm 0.42$ & $198 \pm 0.002$ \\
\hline Rhizopus PR 61 & $27.46 \pm 00$ & - & $1.47 \pm 0.001$ & $93 \pm 0.002$ \\
\hline PR 63* & $61.66 \pm 0.20$ & - & $6.26 \pm 0.02$ & $223 \pm 0.001$ \\
\hline Aspergillus PR 64 & $72.06 \pm 0.27$ & $1.4 \pm 0.065$ & $11.84 \pm 0.02$ & $220 \pm 0.001$ \\
\hline Aspergillus $\mathrm{PR} 65$ & $69.42 \pm 1.55$ & $0.975 \pm 0.001$ & $8.91 \pm 0.02$ & $222 \pm 0.03$ \\
\hline Aspergillus PR 66 & $71.10 \pm 0.18$ & $1.02 \pm 0.01$ & $13.29 \pm 0.046$ & $153 \pm 0.010$ \\
\hline Aspergillus PR 70 & $68.96 \pm 0.47$ & $0.510 \pm 00$ & $5.68 \pm 0.03$ & $193 \pm 0.001$ \\
\hline PR $75^{*}$ & $73.2 \pm 0.18$ & $0.769 \pm 0.001$ & $9.28 \pm 0.011$ & $205 \pm 0.010$ \\
\hline PR 76* & $68.29 \pm 0.54$ & $0.351 \pm 0.001$ & $3.35 \pm 0.005$ & $188 \pm 0.007$ \\
\hline Aspergillus $\mathrm{PR} 78$ & $68.38 \pm 1.05$ & $1.6 \pm 0.02$ & $17.32 \pm 0.05$ & $185 \pm 0.007$ \\
\hline Penicillium PR 79 & $72.14 \pm 0.20$ & $0.98 \pm 0.001$ & $7.28 \pm 0.09$ & $165 \pm 0.005$ \\
\hline PR 80* & $61.60 \pm 0.28$ & - & $3.39 \pm 0.011$ & $205 \pm 0.010$ \\
\hline PR $81^{*}$ & $66.36 \pm 0.47$ & $0.509 \pm 0.002$ & $8.46 \pm .429$ & $187 \pm 0.001$ \\
\hline Aspergillus PR 87 & $65.26 \pm 0.17$ & $0.536 \pm 0.002$ & $9.03 \pm 0.02$ & $176 \pm 0.010$ \\
\hline Aspergillus $\mathrm{PR} 88$ & $67.41 \pm 00$ & $0.389 \pm 0.001$ & $7.28 \pm 0.002$ & $165 \pm 0.001$ \\
\hline PR $89^{*}$ & $84.11 \pm 0.81$ & $0.834 \pm 0.002$ & $11.74 \pm 0.24$ & $186 \pm 0.002$ \\
\hline Penicillium PR 93 & $77.40 \pm 0.35$ & $0.147 \pm 0.001$ & $7.28 \pm 0.020$ & $167 \pm 0.010$ \\
\hline Aspergillus $\mathrm{PR} 94$ & $64.08 \pm 0.45$ & - & $2.37 \pm 0.020$ & $167 \pm 0.010$ \\
\hline Penicillium PR 98 & $66.87 \pm 0.47$ & $0.160 \pm 0.001$ & $8.91 \pm 0.020$ & $175 \pm 0.010$ \\
\hline Penicillium PR 102 & $59.83 \pm 0.57$ & - & $7.06 \pm 0.020$ & $180 \pm 0.010$ \\
\hline PR 104* & $86.93 \pm 0.20$ & $1.05 \pm 0.01$ & $9.97 \pm 0.170$ & $201 \pm 0.010$ \\
\hline Penicillium PR 105 & $69.73 \pm 0.27$ & - & $6.41 \pm 0.020$ & $217 \pm 0.001$ \\
\hline Penicillium PR 106 & $65.58 \pm 0.57$ & - & $7.37 \pm 0.020$ & $196 \pm 0.010$ \\
\hline Aspergillus PR 109 & $63.16 \pm 0.36$ & - & $3.02 \pm 0.020$ & $174 \pm 0.003$ \\
\hline Penicillium PR 110 & $68.72 \pm 0.37$ & $0.298 \pm 0.001$ & $6.19 \pm .0110$ & $194 \pm 0.020$ \\
\hline Aspergillus PR 113 & $62.82 \pm 0.15$ & $1.04 \pm 0.13$ & $12.10 \pm 0.260$ & $177 \pm 0.020$ \\
\hline
\end{tabular}

Each value is the mean \pm standard deviation ${ }^{\mathrm{a}} ; \%$ scavenging activity; ${ }^{\mathrm{b}} \mathrm{mg} / \mathrm{ml}$ of culture; ${ }^{\mathrm{c}} \mathrm{mg} ;-$ no activity; ${ }^{*}$ unidentified

to have a convenient, fast, and universal method for overall quantification of antioxidant efficiency of the natural materials. However, such a test yet has to be developed. A total antioxidant activity assay using one chemical reaction seems to be rather unrealistic, yet there are numerous published methods claiming to measure total antioxidant activity in vitro. In the present study, different assays were employed to assess the comparative antioxidant potential of the fungal extracts. The selected strains giving quantitatively sufficient
DPPH activity were found to be having sufficient reducing power thus complementing each other. However, some strains lacked reducing power. Scavenging potential and reducing power of these extracts are dependent upon their unique phenolic structure, number and position of the hydroxyl groups and their ability to donate hydroxyl groups as well as in which form these phenolic compounds are present, i.e. free or bound form (Zhao et al., 2006 and Sultana et al., 2007). Another probable reason for their lack of reducing power may be that 
Table 2. Comparison between different antioxidant assays as represented by correlation coefficient $(r)(n=51)$.

\begin{tabular}{llll}
\hline & DPPH Assay & $\begin{array}{l}\text { Reducing } \\
\text { power }\end{array}$ & TPC \\
\hline Reducing power & 0.071 & & \\
TPC & 0.178 & 0.541 & \\
Biomass & 0.239 & 0.240 & 0.334 \\
\hline
\end{tabular}

polyphenols present in these fungi are not efficient reducing agent for ferrous iron due to some steric hindrance .On the other hand free hydroxyl groups in phenolic compounds may easily scavenge free radicals (Wong et al., 2006).

Proper correlation could not be established in polyphenolic content and antioxidant activities which may be due to that $\mathrm{FC}$ reagent is not specific for just polyphenols as it can be reduced by many nonphenolic compounds such as vitamins $\mathrm{C}, \mathrm{Cu}$ (I) etc. Various others workers have also reported the poor specificity of the assay (Sultana et al., 2007 and Wonget al., 2006). Further, variation in correlation coefficient among different antioxidant assays indicates that a single assay is not sufficient to evaluate the total antioxidant activity (Table 2). Moreover, the polyphenolic contents of the fungi may not act as an index of their antioxidant activity as they possess many different enzymes, such as catalase and superoxide dismutase and many others components such as glutathione which may be responsible for antioxidant activity (Rapp et al.,1973 and Pócsi et al.,2004). The mechanism in fungi may be different from plants and it may become clearer in near future. Still, if comparison is done with standard ascorbic acid, fungal strains are equally good which proves that further study of such fungal isolates will be beneficial as they are yielding high amount of active components showing comparable good antioxidant activity.

\section{Conclusion}

Our results suggest that the fungi might produce more bioactive compounds apart from antibiotics and these findings will facilitate further studies to gain better understanding of bioactive metabolites production in fungi, which will be helpful in the biotechnological mass production of bioactive metabolites in near future. The results from in vitro experiment including DPPH radical scavenging activity, reducing power method demonstrate that the different soil fungi may have significant antioxidant activities and phenolic compounds, which are responsible for their potential for different biological activities. If positive physiological properties and the non-toxicity of the antioxidant compounds of the fungi is proven these could be suggested as a possible natural sources of antioxidants for incorporation into some food products and supplements to prevent many free radical mediated diseases and the health of consumers. Further investigations are needed to discover bioactive constituents in the fungal isolates and different antioxidant activity of fungi and component analysis should be studied in the near future.

\section{ACKNOWLEDGEMENTS}

The CSIR grant (90(0035)/04/EMR-II) sanctioned to Dr. D.S. Arora was helpful in carrying out this research work.

\section{REFERENCES}

Aljadi, A.M. and Kamaruddin, M.Y. (2004). Evaluation of the phenolics contents and antioxidant capacities of two Malaysian floral honeys. Food Chem., 85: 513-518.

Amarowicz, R., Pegg, R.B., Moghaddam, P.R.,Barl, B. and Weil, J.A. (2004). Free radical scavenging capacity and antioxidant activity of selected plants species from the Canadian prairies. Food Chem., 84:551-562.

Bounatirou, S., Smiti, S., Miguel, M.G., Falerio, L., Rejeb, M. N., Neffati,M., Casto, M.M., Figueiredo, A.C., Barroso, J .G. and Pedro, L .G. (2007). Chemical composition, antioxidant and antibacterial activities of the essential oils isolated from Tunisian Thymus capitatus Hoff. et link. Food Chem., 105: 146-155.

Chang, L.W., Yen, W.J., Huang S.C. and Duh, P. D. (2002). Antioxidant activity of sesame coat. Food Chem., 78: 347354.

Huang, D.J., Chen, H.J., Hou, W. C., Lin, C.D. and Lin, Y.H. (2006). Sweet potato (Ipomoea batatas (L.) Lam 'Tainong $57^{\prime}$ ) storage root mucilage with antioxidant activities in vitro. Food Chem., 98: 774-781.

Huang, W.Y., Cai, Y.Z., Hyde, K.D., Corke, H. and Sun, M. (2007). Endophytic fungi from Nerium oleander L (Apocynaceae): main constituents and antioxidant activity. World J Microbiol Biotechnol., 23:1253-1263.

Kumar, A. and Chattopadhaya S. (2007). DNA damage protecting activity and antioxidant potential of pudina extracts. Food Chem., 100:1377-1384.

Lee, K.I. and Yen, S.B. (2006). Hispidin analoges from mushrooms Inonotus xeranticus and their free radical scavenging activity. Bio Med Chem Lett., 16: 2376-2379.

Mathew, S. and Abraham, T.E. (2006). Studies on the antioxidant activities of cinnamon (Cinnamomum verum) bark extracts, through various in vitro models.Food Chem., 94:520-528.

Miller, M. J., Diplock, A. T. and Rice-Evans, C.A . (1995). Evaluation of the total antioxidant activity as a marker of the deterioration of apple juice on storage. J. Agric. Food Chem., 43: 1794-1801.

Mokbel, M. S. and Hashinaga, F. (2006). Evaluation of the antioxidant activity of extracts from buntan (Citrus grandis Osbeck) fruit tissues. Food Chem., 94: 529-534.

Moon, B.S., Ryoo, I.J., Yun, B. S., Bae, K.S., Lee, K.D.,Yoo, I.D. and Kim, J.P.(2006) .Glyscavins A,B and C,new phenolic glycoside antioxidants produced by a fungus Mycelia sterilia F020054. J. Antibiot., 59: 735-739.

Pócsi, I., Prade, RA. and Penninckx, M.J. (2004). Glutathione, altruistic metabolite in fungi. Adv. Microb. Physiol. , 49: 176.

Rapp, U., Adams, W.C. and Miller, R.W. (1973). Purification 
of superoxide dismutase from fungi and characterization of the reaction of the enzyme with catechols by electron spin resonance spectroscopy. Can. J. Biochem., 51: 158-171.

Rios, M.F., Pajan, C.M.G., Galan, R.H., Sanchez,A.J.M. and Callado, I.G. (2006). Synthesis and free radical scavenging activity of a novel metabolite from the fungus Colletotrichum gloeosporioides. Bioorg. Med. Chem. Lett., 16: 5836-5839.

Robbins, R.J. (2003) .Phenolic acids in foods: An overview of analytical methodology. J.Agri. Food Chem., 51:2866-2887.

Singleton,V.L., Ortofehr, R. and Lamuela-Raventos, R.M.(1999). Analysis of total phenols and other oxidation substrate and antioxidants by means of Folin-Ciocalteau reagent. Methods Enzymol., 299:152-178.

Song, T.Y. and Yen, G.C. (2002). Antioxidant properties of Antrodia camphorata in submerged culture. J. Agri. Food chem., 50: 3322-3327.

Sultana,B., Anwar,F. and Przybylski, R. (2007). Antioxidant activity of phenolic components present in barks of Azadirachta indica, Terminalia arjuna, Acacia nilotica and Eugenia jambalana Lam.trees. Food Chem., 104: 11061114.
Tiwari, O. P. and Tripathi, Y. (2007). Antioxidant properties of different fractions of Vitex negundo Linn. Food Chem., 100 :1170-1176.

Valentao, P., Fernandes, E., Carvalho, F., Andrade, P.B., Seabra, R.M. and Bastos,M.L. (2002). Antioxidant activity of Hypericum androsaemum Infusion: scavenging activty against superoxide radical, hydroxyl radical and hypochlorous acid. Biol. Pharm. Bull., 25: 1320-1323.

Virtanen,T., Pihlanto, A., Akkanen, S. and Korhonen, H. (2007). Development of antioxidant activity in milk whey during fermentation with lactic bacteria. J. Applied Microbiol., 102:106-115.

Wong, S. P., Leong,L.P. and Koh, J. H. W. (2006). Antioxidant activities of aqueous extracts of selected plants. FoodChem., 99: 775-783.

Zhao, G.R.,Xiang, Z.J.,Ye,T.X. Yaun, J.Y. and Guo, X.Z. (2006). Antioxidant activities of Silvia miltiorrhiza and Panax notoginseng. Food Chem., 99:767-774.

Zielinski, H. and Kozlowska, H. (2000). Antioxidant activity and total phenolics in selected cereal grains and their different morphological fractions. J. Agric. Food Chem., 48: 20082016. 